\title{
Identifying Beatles songs from their chord progressions: New evidence of the effect of specialized harmonic familiarity, melodic cues, and transposition on the identification of songs from chord progressions
}

\author{
Tuire Kuusi ${ }^{1}$ \\ Ivan Jimenez ${ }^{2 \dagger}$ \\ Matthew D. Schulkind ${ }^{3}$ \\ ${ }^{1}$ Sibelius Academy, University of the Arts Helsinki, Finland \\ ${ }^{2}$ Department of Psychology, Amherst College, Amherst, MA, USA \\ † Corresponding author: ivan.jimenez.rodriguez@uniarts.fi \\ Published 16 December 2021; https://doi.org/10.18061/FDMC.2021.0035 \\ Author video presentation and/or other conference material: https://doi.org/10.17605/OSF.IO/M4ZVJ
}

\begin{abstract}
Listener and musical factors influence the identification of songs from chord progressions. Having played and being able to write out the chords of the target song from longterm memory (hereafter, specialized harmonic familiarity) facilitate the identification of jazz standards from their chord progressions among Jazz musicians. Additionally, both musicians and non-musicians find it easier to identify popular songs and pieces of classical music from chord progressions when stimuli are played using piano tones as opposed to Shepard tones, an effect that may be at least partially driven by the melodic ambiguity that Shepard tones create. The present study investigated whether similar and additional effects can be observed under different experimental conditions. Adopting a gating paradigm, this new study tested the ability of 303 Beatles fans to identify four well-known Beatles songs from chord progressions played using piano tones. Results confirm previous findings regarding the effect of melodic cues, provide some evidence of the effect of transposition, and show that specialized harmonic familiarity has an effect on the identification of songs from chord progressions for repertoires other than jazz standards and among listeners who have very diverse musical backgrounds.
\end{abstract}

KEYWORDS: memory for harmony, popular music, Beatles, transposition, gating paradigm

\section{Introduction}

There is plenty of research showing that listeners, regardless of their musical training, can identify pieces of music from the melody. Identification is possible even if the musical material is manipulated in one way or another (for a review, see Halpern \& Bartlett, 2010). Some studies also indicate that timbre may facilitate the identification of pieces of music, especially if the excerpts are very short (Krumhansl, 2010;
Schellenberg, Iverson, \& McKinnon, 1999). There has been considerably less research on the contribution of harmony to the identification of music, which might be due to the fact that aural focus on harmony is not easy because of the extra-harmonic features affecting in perception (Cullimore, 1999; Farbood, 2012; Halpern, 1984; Mélen \& Deliège, 1995; Williams, 2005).

It has been shown that various aspects of musical training sharpen listeners' attention to harmony (Farbood, 2012; Norgaard, 2017; Sears, Caplin, \& McAdams, 2014; Williams, 2005). For example, identifying well-known pieces of classical music as well as pop and rock songs from the harmony appears to be easier for musicians than for non-musicians (Jimenez \& Kuusi, 2018; Jimenez, Kuusi, \& Doll, 2020), and having played the target piece and being able to retrieve the chord labels of the pieces from the long-term memory (referred to as "specialized harmonic familiarity") facilitate the identification of jazz standards from their chord progressions among jazz musicians (Jimenez \& Kuusi, 2020). Additionally, identifying popular songs and pieces of classical music from chord progressions has been shown to be easier when stimuli are played using piano tones as opposed to Shepard tones, an effect that may be at least partially driven by the melodic ambiguity that Shepard tones create (see, e.g., Jimenez \& Kuusi, 2018).

The present study aimed at expanding our understanding of listeners' ability to identify songs from chord progressions. Specifically, we tested whether specialized harmonic familiarity has an effect on the identification of songs from chord progressions for repertoires other than jazz standards and among listeners who have more diverse musical backgrounds than the professional jazz musicians and advanced jazz students previously tested. Therefore, we decided to use well-known Beatles songs and a large number of 
non-professional participants who were familiar enough with the target songs to name all of them from commercial recordings. We, further, tested the following musical factors: melodic cues, transposition, and the number of chords.

\section{Methods}

Altogether 303 Beatles fans (246 male, 54 female, and 3 other; mean age $=37.2, S D=14.4$ ) participated. Males were overrepresented, but we do not know of any research showing that gender has any effect on harmonic awareness. The task was to identify four well-known Beatles songs from chord progressions (up to 16 chords from the beginning of the song) played using piano block chords. The songs were chosen according to their popularity (number of Last.fm listeners), harmonic rhythm (isochronous) and chord duration (IOI between 1.95 and 2.925 seconds), harmonic uniqueness (not only I, IV and V), and chord texture (not identical to the texture of our chordal stimuli). The four Beatles that fulfilled our selection criteria were "Let It Be," "Lucy in the Sky with Diamonds," "While My Guitar Gently Weeps," and "A Day in the Life." We refer to these four songs in the rest of the text as LET, LUCY, GUIT, and DAY, respectively.

Four different versions of each chord progression were prepared: (1) melodic cues in top voice and original key, (2) melodic cues in top voice but transposed, (3) no melodic cues but original key, (4) no melodic cues and transposed. The top voice in the melodic-cues version was composed to represent the most prominent pitches of the original vocal melody. The transposed version was always a tritone down from the original key. The chord progressions were recorded with a Steinway grand piano sound using GarageBand software (Apple Inc.). The duration of the chords matched the harmonic tempo from the best well-known commercial recording of each song. Each participant heard all four songs and the conditions, but only one condition per song.

A gating paradigm was used: Chord progressions were presented multiple times, increasing the number of chords with each new presentation. We created a gating sequence with five conditions, using $2,3,4,8$, and 16 chords from the beginning of each song. The participants were encouraged to listen to the excerpts until they were sure that they had identified the song, and then provide the name of the song or words from the lyrics as a response. After finishing the gating experiment, the participants heard the songs as 15- second excerpts from commercial recordings and were asked to name them and to provide information about whether they had ever played the chords of the song in question. At the end of the experiment the participants were asked to write from memory (using chord symbols or other types of chord labels) the first six chords of the songs used in the experiment.

\section{Results}

Song identification from chords was possible even for non-professional listeners. As little as two chords was enough for song identification in some cases. LET was the most easily identified while DAY was the most difficult (Table 1).

Table 1: Number of participants who identified the songs from their chord progressions.

\begin{tabular}{lcc}
\hline $\begin{array}{l}\text { Target } \\
\text { piece }\end{array}$ & $\begin{array}{c}\text { Participants who } \\
\text { identified the song }\end{array}$ & $\begin{array}{l}\text { Participants who did not } \\
\text { identify the song }\end{array}$ \\
\hline LET & 259 & 44 \\
GUIT & 198 & 105 \\
LUCY & 109 & 194 \\
DAY & 91 & 212 \\
\hline
\end{tabular}

We were interested in the extent to which identification was influenced by the participant's specialized harmonic familiarity with the songs, that is, whether they had played the target piece and how well they had been able to write the chords of the song from memory. We identified the following three participant groups: PW (P standing for Play and $\mathrm{W}$ for Write), comprising participants who reported having played the target piece at least three times and were able to write the chords; PnW (Play, not Write), those who reported having played the target piece at least three times but were unable to write the chords; and $\mathrm{nPnW}$ (neither Play nor Write). We did not use the nPW group because there were very few cases in which the participant was able to write the chords without having played the piece.

In order to analyze the number of chords needed for identification, we used conditional testing, the conditions being 2, 3, 4, 8, and 16 chords. The conditional ID percentage (ID\%) was calculated separately for each target piece and participant group. The ID\% (shown in Figure 1) indicates the number of 
participants who identified the target piece at the condition of $\mathrm{x}$ chords as a percentage of all participants who had not yet identified the piece. As the figure indicates, having played a piece's chords and being able to write them appeared to help with the identification of the piece from its chord progression, at least to some extent.

LET Conditional ID\%

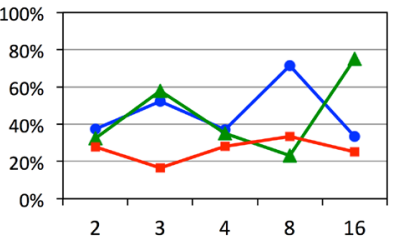

LUCY Conditional ID\%

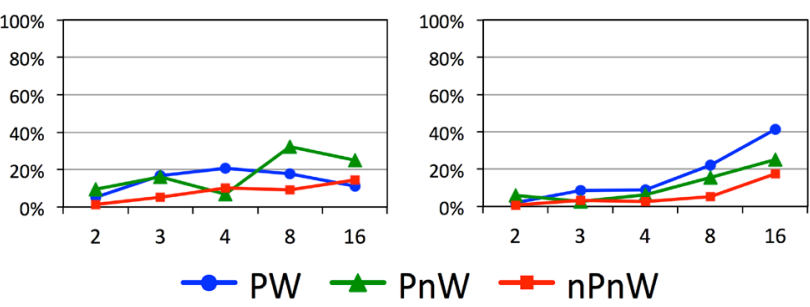

Figure 1: The conditional ID\%s for the three groups of participants, separately for each target piece. The horizontal axis shows the conditions.

An independent-samples Kruskal-Wallis test showed that there were statistically significant differences between the participant groups for LET ( $p$ $=.046)$, and marginally significant differences for GUIT ( $p=.056$ ) but not for the other two pieces. The pairwise comparisons showed that the differences were between participant groups $\mathrm{PW}$ and nPnW. The standard test statistics were as follows: PW$\mathrm{nPnW}($ LET $)=2.406, p=.048$ and $\mathrm{PW}-\mathrm{nPnW}($ GUIT $)$ $=2.404, p=.049$.

Figure 1 also shows that adding more chords did not systematically increase the ID\%. Instead, there are conditions at which identification seems to be easier than at some other points. DAY(16), for example, seems to be a point of identification in all the participant subgroups, and the identification could be attributable to the distinctive chord succession bVII(add\#11)-vi. In the case of GUIT, specialized harmonic familiarity had a general effect, but the identification points were seemingly similar for all groups of participants. As the figures show, there was a small peak at 3 chords and a higher one at 8 chords, both of which were most likely related to the occurrence of the major IV chord (D chord in A minor), which contains a raised $6^{\text {th }}$ scale-degree (F\# in A minor).

We also analyzed the effect of transposition and melodic cues on the participants' ability to identify each song. We found that the chord progressions from LET and GUIT played in the original key were identified more frequently than the transposed chord progressions. On the other hand, chord progressions from GUIT, LUCY and DAY without melodic cues were identified less frequently than progressions with melodic cues. In sum, the results show that the original key and melodic cues tended to facilitate identification, but the effect depended on the song (for details of all analyses, see Kuusi, Jimenez, \& Schulkind, 2021).

\section{Discussion and Conclusions}

We showed that it is possible for listeners other than professional musicians to identify pieces of music from very little harmonic information, even without correct melodic cues and heard in a key that is distant from the original. The number of chords needed for identification was not the same for all pieces or participants, varying from as little as two to as many as 16. The extent to which adding chords made the songs easier to identify also varied. Of the participant background variables, the specialized harmonic familiarity (the participants' ability to write the chord labels from memory and their having played the target piece) was the only one that had an effect on identification. Generally, the results are in line with our earlier findings (Jimenez \& Kuusi 2018, 2020), although there was variation between the individual songs.

Some of the identification patterns are consistent with the view that harmonic uniqueness facilitates song identification (Coker, Knapp, \& Vincent, 1997). However, harmonic uniqueness did not seem to be required or sufficient for the identification of these songs. For instance, $58 \%$ of the participants were able to identify LET from just two chords, I-V, one of the most common chord successions in tonal music. Moreover, relatively distinctive harmonic events such as the shift from emphasizing $\mathrm{C}$ major to emphasizing its relative minor in LET, the shift from the key of A minor to its parallel major in GUIT, and the modulation from A Mixolydian to Bb Lydian in LUCY did not increase identification rates compared to other 
less distinctive harmonic events in the progressions. Future research could focus on the time course of the identification of songs from the harmony, and specifically the potential role of harmonic uniqueness in that process.

In our study, transposition seemed to affect the identification of LET and GUIT, but not of the other two songs. We do not know whether our participants had been exposed to only one transposition (which could explain the result) of the songs - especially if they had played the songs themselves. Correct melodic cues tend to help identification, and this was the case in our study, except for LET. Although the versions with and without melodic cues were created following the same rules, these rules seem to have resulted in the uncued version of LET still having melodic cues, the melodically downgraded upper voice of the in-cued LET started with scale-degrees 3-2-1 and happened to be similar to the original vocal melody for the fifth (I), sixth (V), seventh (IV), and eighth chords (I) of the song.

One possible factor affecting the identification of LET could have something to do with the popularity of the song. Participants in an experiment concerning famous Beatles songs would probably expect to hear "Let It Be". If a participant was expecting to hear the words "Let it be" before hearing the first chord, and tried mentally to sing "Let it be" on top of the chords to see if the melody matched the stimuli, that top-down strategy would be affected by downgraded melodic cues to a lesser extent than a bottom-up strategy that allowed him or her to rely on the melodic features of the stimuli only in activating long-term-memory traces of the song (e.g., Schellenberg, Iverson, \& McKinnon, 1999). This strategy could have been strengthened by the timbral similarity between the original and the stimulus played on piano.

In sum, our study shows that there are various factors affecting song identification from the harmony. As correct melodic cues were found to help identification, the roles of transposition and specialized harmonic familiarity were not as clear, and our results imply that they may only have an impact on songs that are relatively easy to identify from their chord progressions. Our chordal stimuli were more similar to the original commercial recordings in LET and GUIT, the two most often identified songs in our experiment, than in LUCY and DAY in terms of timbre (piano tones) and texture (one unembellished block chord per chord change). Therefore, our findings indicate that specialized harmonic familiarity and transposition are more likely to affect the identification of songs from chords when the extra-harmonic similarity between the chordal stimuli and the original is relatively high. One possible explanation for this is that participants who are very familiar with a song and its harmony have a higher likelihood of using top-down identification strategies such as singing the melody on top of the chord or recollecting the chord labels of the song. Close transposition and extra-harmonic similarity between stimuli and the original could also facilitate the success of such top-down strategies. We must point out, however, that we only tested four songs representing a particular segment of popular music, and that further testing of these ideas would require a larger set of songs. Future research is needed to assess the effect of these and other aspects of musical and participant factors on the identification of songs from harmony.

\section{Acknowledgements}

We thank the editors of the DocMus Research Publications for kindly permitting us the possibility to publish this shortened version of the original article.

\section{References}

Coker, J, Knapp, B., \& Vincent, L. (1997). Hearin' the changes: Dealing with unknown tunes by ear. [Rottenburg:] Advance music.

Cullimore, J. R. (1999). Harmonic hierarchies as distinctive abstractions that listeners may derive from musical surface structure. MA Thesis, Queen's University at Kingston (Canada).

Farbood, M.M. (2012). A parametric, temporal model of musical tension. Music Perception: An Interdisciplinary Journal 29(4), 387-428. https://doi.org/10.1525/mp.2012.29.4.387

Halpern, A. R. (1984). Perception of structure in novel music. Memory \& Cognition 12(2), 163-70. https://doi.org/10.3758/BF03198430

Halpern, A. R., \& Bartlett, J. C. (2010). Memory for melodies. In M. R Jones. R.R. Fay, \& A. N. Popper (Eds.), Music Perception, 233-58. New York: Springer. https://doi.org/10.1007/978-1-4419-6114-3_8

Jimenez, I. \& Kuusi, T. (2018). Connecting chord Progressions with specific pieces of music. Psychology of Music 46(5), 716-33. https://doi.org/10.1177/0305735617721638

Jimenez, I. \& Kuusi, T. (2020). What helps jazz musicians name tunes from harmony? A study of the effect of general and specific Work with harmony on the ability to identify music from chord progressions. 
Psychology of Music 48 (2), 215-31. https://doi.org/10.1177/0305735618793005

Jimenez, I., Kuusi, T., \& Doll, C. (2020). Common chord progressions and feelings of remembering. Music \& Science $3, \quad 1-16$. https://doi.org/10.1177/2059204320916849

Krumhansl, C. L. (2010). Plink: "Thin slices" of music. Music Perception: An Interdisciplinary Journal 27(5), 337-54. https://doi.org/10.1525/mp.2010.27.5.337

Kuusi, T., Jimenez, I., Schulkind, M. (2021). Revisiting the effect of listener and musical factors on the identification of music from chord Progressions. In J. Ojala and L. Suurpää (Eds.) Musical Performance in Context: A Festschrift in Celebration of Doctoral Education at the Sibelius Academy. DocMus Research Publications 17. Helsinki: Sibelius Academy.

Mélen, M., \& Deliège, I. (1995). Extraction of cues or underlying harmonic structure: Which guides recognition of familiar melodies? European Journal of Cognitive Psychology 7(1), 81-106. https://doi.org/10.1080/09541449508520159

Norgaard, M. (2017). Descriptions of improvisational thinking by developing jazz improvisers. International Journal of Music Education 35(2), 259-71. https://doi.org/10.1177/0255761416659512

Schellenberg, E. G., Iverson, P., \& Mckinnon, M. C. (1999). Name that tune: Identifying popular recordings from brief excerpts. Psychonomic Bulletin \& Review 6(4), 641-46. https://doi.org/10.3758/BF03212973

Sears, D., Caplin, W. E., and McAdams, S. (2014). Perceiving the classical cadence. Music Perception: An Interdisciplinary Journal 31(5), 397-417. https://doi.org/10.1525/mp.2014.31.5.397

Williams, L. R. (2005). Effect of music training and musical complexity on focus of attention to melody or harmony. Journal of Research in Music Education 53(3), 210-21. https://doi.org/10.1177/002242940505300303 